\title{
Characterization benches for neutrino telescope Optical Modules at the APC laboratory
}

\author{
Avgitas Theodore $^{\mathrm{a}}$, Creusot Alexandre ${ }^{\mathrm{b}}$, and Kouchner Antoine for the KM3NeT collaboration \\ Laboratoire APC - Université Paris Diderot - Paris VII, France
}

\begin{abstract}
As has been demonstrated by the first generation of neutrino telescopes Antares and IceCube, precise knowledge of the photon detection efficiency of optical modules is of fundamental importance for the understanding of the instrument and accurate event reconstruction. Dedicated test benches have been developed to measure all related quantities for the Digital Optical Modules of the KM3NeT neutrino telescope being currently deployed in the Mediterranean sea. The first bench is a black box with robotic arms equipped with a calibrated single photon source or laser which enable a precise mapping of the detection efficiency at arbitrary incident angles as well as precise measurements of the time delays induced by the photodetection chain. These measurement can be incorporated and compared to full GEANT MonteCarlo simulations of the optical modules. The second bench is a $2 \mathrm{~m} \times 2 \mathrm{~m} \times 2 \mathrm{~m}$ water tank equipped with muon hodoscopes on top and bottom. It enables to study and measure the angular dependence of the DOM's detection efficiency of the Cherenkov light produced in water by relativistic muons, thus reproducing in situ detection conditions. We describe these two benches and present their first results and status.
\end{abstract}

\section{Introduction}

Two major neutrino projects take place in the depths of the Mediterranean Sea. The Antares neutrino telescope already provides the scientific community with data while the next generation detector KM3NeT would be a major step towards the understanding of fundamental neutrino properties and will open a new window for the study of astrophysical objects through neutrino detection.

The basic observation units of these telescopes are the Optical Module (Antares) and the Digital Optical Module (KM3NeT). The knowledge of their operational characteristics is of major importance for the efficiency of these telescopes. In this context the APC Laboratory has already constructed a fully functional test bench, implementing a black box with a wide variety of light sources, while the development of a second test bench has just finished, combining a water tank with a hodoscope for muon Cherenkov detection. With this two test benches the APC provides a full calibration system for OMs and DOMs.

\footnotetext{
a e-mail: avgitas@apc.in2p3.fr

b e-mail: creusot@apc.in2p3.fr
}

This is an Open Access article distributed under the terms of the Creative Commons Attribution License 4.0, which permits unrestricted use, distribution, and reproduction in any medium, provided the original work is properly cited. 

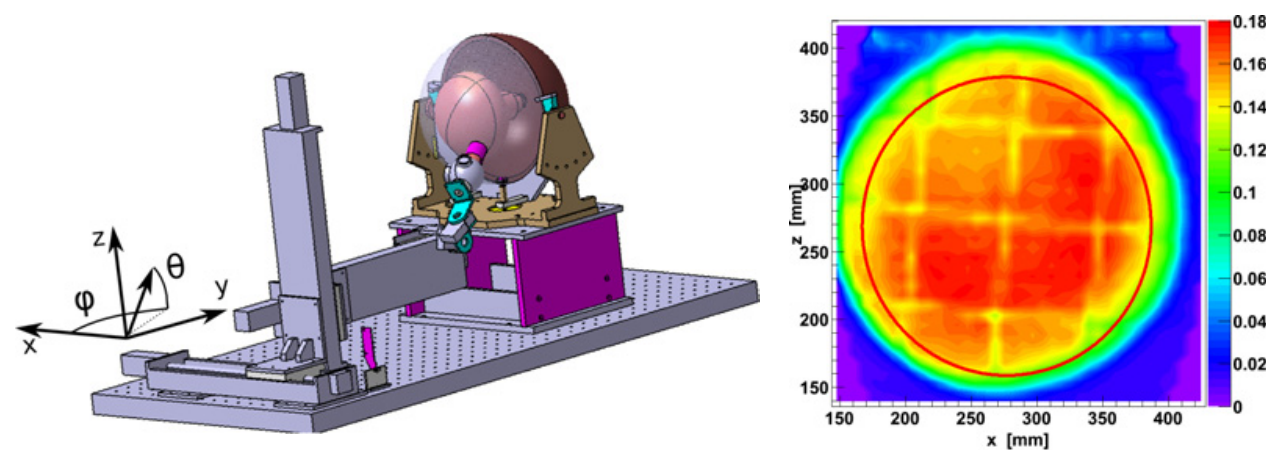

Figure 1. In the left, scheme of the content of the black box. The origin of the coordinate system stands at the corner of the table. The emission direction of the photons is completely defined by the 2 angles $\theta$ (zenith) and $\phi$ (azimuth). The origin $\left(\theta\right.$ and $\left.\phi=0^{\circ}\right)$ corresponds to normal incidence when the light source is facing the PMT in its center. In the right, scan of the photocathode surface of an Antares OM. 11449 points of measurement have been taken. The points are positioned regularly on the optical module surface with a separation of $0.75^{\circ}$. For each point, $10^{6}$ triggers were saved (statistical error of $1 \%$ ).

\section{Black box}

\subsection{Test bench set up}

The black box is a parallelepiped $2 \mathrm{~m}$ long, $1 \mathrm{~m}$ large and $1 \mathrm{~m}$ high. It is equipped with an Antares Optical Module (OM [1]), a light source, 5 motor arms and their respective structures (see Fig. 1). All the holding structures are kept fixed on an optical table. The communications with the components within the box are allowed by several junction panels.

The 5 motors installed in the box allow choosing the position $(x, y, z)$ of the light source and the direction of the emission $\left(v_{\mathrm{x}}, v_{\mathrm{y}}, v_{\mathrm{z}}\right)$ of the photons. The motor step size for the position and the direction is smaller than $0.01 \mathrm{~mm}$ and 1 arcsecond, respectively. The motors are driven via the LabVIEW software installed in a computer.

The light source, an integrating sphere, has been developed at APC [2]. It consists of a sphere fitted with a matrix of Light Emitting Diode (LED) centered on $\lambda=378 \mathrm{~nm}$. A small fraction of the light of the diodes can escape by a small hole made in the sphere, allowing to have a really weak and calibrated light emission. An additional collimator selects the direction of emission of the photons within a cone of aperture half-angle $5.4^{\circ}$. This device allows reproducing with good accuracy the experimental environment, i.e. a majority (95\%) of one photon pulses. One of the main characteristics of the sphere is that the power escaping through a hole is proportional to the area of the hole allowing an absolute calibration of the device.

The command voltage of the OM is set so that the PMT gain is nominal, i.e. the signal from a detected photon has an amplitude of about $45 \mathrm{mV}$. The anode signal is sent to the acquisition system $[3,4]$. Above a threshold of $1 / 3$ pe, the signal charge is integrated over $80 \mathrm{~ns}$. Knowing the number of photons exiting the sphere for each LED trigger, it is straightforward to estimate the absolute efficiency of detection of the OM.

\subsection{Results}

For the scan presented in this article, the direction of emission of the photons is kept colinear to $y$ (see left plot of Fig. 1) and the position of the light source follows the OM surface (steps of $0.75^{\circ}$ ) in order to simulate a parallel beam of light. The results are shown in the right plot of Fig. 1. The color scale represents the absolute detection efficiency of the OM photocathode. An average detection efficiency 

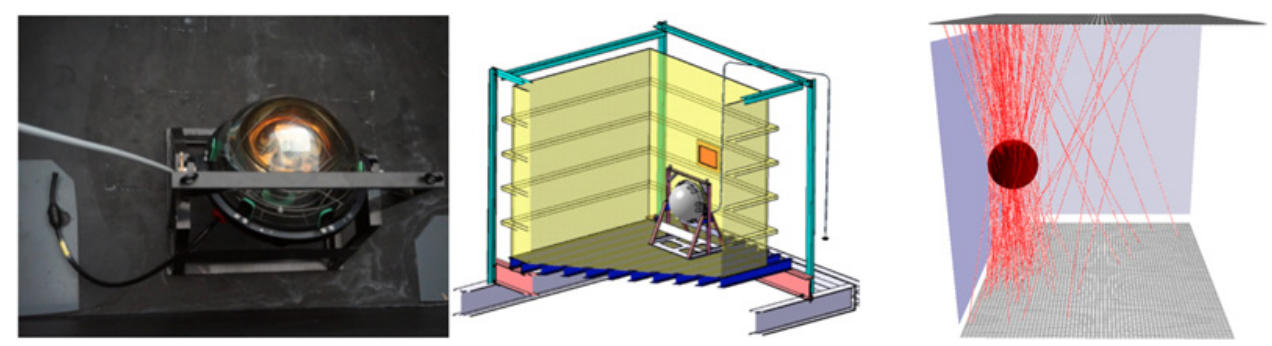

Figure 2. The OM attached with clamps on its base (left). An artistic representation of the OM inside the tank (center). Visualization of the muon tracks (right). The grids on top and bottom represent the hodoscope. The tracks crossing outside the $\mathrm{OM}$ are plotted because only time correlation criteria are applied thus extracting multiple track candidates per OM event.

value of $15.8 \%$ has been found within the red circle representing a photocathode of $500 \mathrm{~cm}^{2}$. The mumetal grid can clearly be seen in the scan as yellow strips of lower efficiency.

\section{APC Water Tank}

For the characterization of the OMs a Water Tank with a Hodoscope, originally developed for LAGUNA/MEMPHYNO [5], is maintained at the facilities of the APC.

\subsection{Test bench Set up}

The photo proof water tank is of cubic shape with edge size of $2 \mathrm{~m}$. It is made out of High Density Polyethylene (HDPE). The inner walls are of black color. Inside the tank the OM is fixed on its base via multiple clamps (see left plot of Fig. 2). The OM support also allows different Zenith angles for the OM that are manually adjusted through a rotor (see center plot of Fig. 2).

\section{Hodoscope}

It consists of four identical scintillation detectors originated from the Track Tracker of the OPERA experiment [6], properly customized to fit the needs of the present set up. Each detector is $2 \mathrm{~m}$ in length, $1.7 \mathrm{~m}$ in width and approximately $1 \mathrm{~cm}$ thick. The scintillator (polystyrene, $2 \% \mathrm{p}$-Terphenyl, $0.02 \%$ POPOP) is arranged in independent strips of $1.6 \mathrm{~m}$ length, $2.6 \mathrm{~cm}$ width and height of $1 \mathrm{~cm}$. The strips are coated with $\mathrm{TiO}_{2}$ as reflective material. An optical fiber collects and guides the photons from the scintillator to the photodetector (an $8 \times 8$ multianode PMT).

Two detectors placed perpendicular to each other form a Matrix of $64 \times 64$ channels, providing an accuracy of $2.6 \mathrm{~cm} \times 2.6 \mathrm{~cm}$ for the $(\mathrm{x}, \mathrm{y})$ positions. One such pair is placed on top of the tank while another one resides below it. A commercial Hamamatsu H8808 PMT is used for the detection of the scintillator signals [7]. A CAEN multichannel high voltage supply is used for the operation of all the PMTs of the Hodoscope.

\section{Hodoscope Read Out}

The 64 PMT anode signals are sent to a MAROC3 chipset [8] equipped with an FPGA card for signal treatment and triggering. The MAROC3 has been designed to amplify individually each channel and to produce, below a common threshold, a trigger. Each trigger is then time stamped and sent to an acquisition computer together with the strip and hodoscope identities. The four cards are adjusted so that 
the channels have similar trigger rates at a threshold level equivalent to $1 \mathrm{pe}$. They are also synchronized though a $40 \mathrm{MHz}$ GPS and a $100 \mathrm{~Hz}$ PPS.

\section{Antares OM set up}

The $\mathrm{OM}$ was placed on its base and inside the empty photo proof tank facing upwards, $\theta=0^{\circ}$. The communication with the $\mathrm{OM}$ is established through its control box, which serves two purposes. It supplies the OM with high voltage and provides an output for the signal. The signal is then delivered, using a $16 \mathrm{~ns}$ RG-58 cable, to a MAROC3 card signal input (channel 0). The threshold of the channel is set at $\sim 0.02 \mathrm{mV}$. For what concerns the OM, the output of the card is the measured charge in ADC units (instead of the strip identity) and the time of the event. The time of this card is also synchronized with that of the hodoscope through the same GPS and PPS. The OM was operated at its nominal voltage.

\subsection{Results}

The acquired data have been used to run a visualization program indicating those particle tracks detected together by the 4 scintillators and the $\mathrm{OM}$ in a time window of $100 \mathrm{~ns}$. This result is presented in the right plot of Fig. 2. Some tracks are obviously not crossing the OM, this could be due to the fact that the program uses time correlation for the events and it happens (rarely) to find two candidate tracks for the same OM event and then plots both.

\section{References}

[1] P. Amram et al. [Antares Collaboration], Nucl. Instrum. Meth. A484 (2002) 369-383

[2] P. Gorodetzky, Sphères intégratrices Brevet France $N^{\circ}$ 06/09088

[3] D. Breton, E. Delagnes, J. Maalmi, Picosecond time measurement using ultra fast analog memories, in proceedings of TWEPP-09, Topical Workshop on Electronics for Particle Physics, Paris, September 2009

[4] D. Breton, E. Delagnes, Fast analog sampler with great memory depth, FR2824177 and US No.6859375 patents

[5] A. Tonazzo et al. [LAGUNA-LBNO Collaboration], Nucl. Part. Phys. Proc. 265-266 (2015) 192-194

[6] T. Adam and others, The OPERA experiment Target Tracker, Nucl. Instrum. Meth. A577 (2007) 523-539

[7] M. Marafini, Physics studies and R\&D towards the MEMPHYS experiment: A water Cherenkov Detector in Europe, Université Paris Diderot, École Doctoral 517, 2011

[8] S. Blin et al. 2010 JINST 5 C12007 